\title{
Chidamide in relapsed or refractory peripheral T cell lymphoma: a multicenter real-world study in China
}

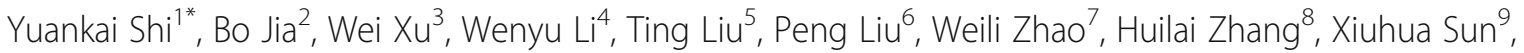
Haiyan Yang ${ }^{10}$, Xi Zhang ${ }^{11}$, Jie Jin ${ }^{12}$, Zhengming Jin ${ }^{13}$, Zhiming Li ${ }^{14}$, Lugui Qiü ${ }^{15}$, Mei Dong ${ }^{1}$, Xiaobing Huang ${ }^{16}$, Yi Luo ${ }^{17}$, Xiaodong Wang ${ }^{16}$, Xin Wang ${ }^{18}$, Jianqiu Wu ${ }^{19}$, Jingyan $\mathrm{Xu}^{20}$, Pingyong $\mathrm{Yi}^{17}$, Jianfeng Zhou ${ }^{21}$, Hongming $\mathrm{He}^{22}$, Lin Liu ${ }^{23}$, Jianzhen Shen ${ }^{24}$, Xiaogiong Tang ${ }^{23}$, Jinghua Wang ${ }^{25}$, Jianmin Yang ${ }^{26}$, Qingshu Zeng ${ }^{27}$, Zhihui Zhang ${ }^{28}$, Zhen Cai ${ }^{12}$, Xiequn Chen ${ }^{29}$, Kaiyang Ding ${ }^{30}$, Ming Hou ${ }^{31}$, Huiqiang Huang ${ }^{14}$, Xiaoling Li ${ }^{32}$, Rong Liang ${ }^{29}$, Qifa Liü ${ }^{33}$, Yuqin Song ${ }^{2}$, Hang Su ${ }^{34}$, Yuhuan Gao ${ }^{35}$, Lihong Liư ${ }^{35}$, Jianmin Luo ${ }^{36}$, Liping Su ${ }^{37}$, Zimin Sun ${ }^{30}$, Huo Tan ${ }^{38}$, Huaqing Wang ${ }^{39}$, Jingwen Wang ${ }^{40}$, Shuye Wang ${ }^{41}$, Hongyu Zhang ${ }^{42}$, Xiaohong Zhang ${ }^{43}$, Daobin Zhou ${ }^{44}$, Ou Bai ${ }^{45}$, Gang Wu ${ }^{46}$, Liling Zhang ${ }^{46}$ and Yizhuo Zhang ${ }^{8}$

\begin{abstract}
The efficacy and safety of chidamide, a new subtype-selective histone deacetylase (HDAC) inhibitor, have been demonstrated in a pivotal phase II clinical trial, and chidamide has been approved by the China Food and Drug Administration (CFDA) as a treatment for relapsed or refractory peripheral T cell lymphoma (PTCL). This study sought to further evaluate the real-world utilization of chidamide in 383 relapsed or refractory PTCL patients from April 2015 to February 2016 in mainland China. For patients receiving chidamide monotherapy $(n=256)$, the overall response rate (ORR) and disease control rate (DCR) were 39.06 and 64.45\%, respectively. The ORR and DCR were 51. 18 and $74.02 \%$, respectively, for patients receiving chidamide combined with chemotherapy $(n=127)$. For patients receiving chidamide monotherapy and chidamide combined with chemotherapy, the median progression-free survival (PFS) was 129 (95\% Cl 82 to 194) days for the monotherapy group and 152 (95\% Cl 93 to 201) days for the combined therapy group ( $P=0.3266$ ). Most adverse events (AEs) were of grade 1 to 2 . AEs of grade 3 or higher that occurred in $\geq 5 \%$ of patients receiving chidamide monotherapy included thrombocytopenia (10.2\%) and neutropenia (6.2\%). For patients receiving chidamide combined with chemotherapy, grade 3 to 4 AEs that occurred in $\geq 5 \%$ of patients included thrombocytopenia (18.1\%), neutropenia (12.6\%), anemia (7.1\%), and fatigue (5.5\%). This large real-world study demonstrates that chidamide has a favorable efficacy and an acceptable safety profile for refractory and relapsed PTCL patients. Chidamide combined with chemotherapy may be a new treatment choice for refractory and relapsed PTCL patients but requires further investigation.
\end{abstract}

Keywords: Chidamide, Peripheral T cell lymphoma, Treatment, Chemotherapy

\footnotetext{
* Correspondence: syuankaipumc@126.com; syuankai@cicams.ac.cn

'Department of Medical Oncology, Beijing Key Laboratory of Clinical Study

on Anticancer Molecular Targeted Drugs, National Cancer Center/Cancer

Hospital, Chinese Academy of Medical Sciences and Peking Union Medical

College, Beijing 100021, China

Full list of author information is available at the end of the article
} 


\section{Letter to the editor}

Peripheral $\mathrm{T}$ cell lymphomas (PTCLs) are a set of rare and highly heterogeneous tumors derived from mature $\mathrm{T}$ cells or natural killer cells and are typically characterized by poor prognosis and aggressive clinical behavior [1]. PTCL accounts for 23 to $26 \%$ of all non-Hodgkin's lymphoma (NHL) in China, which is significantly higher than the rates in Western countries [2, 3]. A consensus has not been reached on standard treatments for PTCL patients, and most commonly used traditional chemotherapy regimens are associated with a poor response [1, 4]. Moreover, a majority of patients may experience disease relapse even if they receive high-dose chemotherapy and autologous stem cell transplantation (ASCT) $[5,6]$

Since 2009, the US Food and Drug Administration (FDA) has approved four new drugs for the treatment of relapsed or refractory PTCL, including the histone deacetylase (HDAC) inhibitors romidepsin and belinostat, the dihydrofolate reductase inhibitor pralatrexate, and the CD30 antibody-drug conjugate brentuximab vedotin for CD30-positive anaplastic large cell lymphoma (ALCL) patients [7, 8].

Chidamide, an innovative new drug independently developed in China, is designed to selectively inhibit the activity of HDAC1, 2, 3, and 10 following oral administration and was approved in December 2014 by the China Food and Drug Administration (CFDA) for the treatment of relapsed or refractory PTCL [9].

The efficacy and safety of chidamide have been demonstrated in a pivotal phase II clinical trial [10], yet further evaluation of its real-world utility is urgently needed. Therefore, we conducted a real-world multicenter efficacy and safety monitoring study to further test the clinical practice value of chidamide in relapsed or refractory PTCL patients in mainland China.

We analyzed 383 patients from April 2015 to February 2016. The cutoff date was February 19, 2016. The methods are shown in Additional file 1. The baseline characteristics of all patients are presented in Additional file 2.

For patients receiving chidamide monotherapy $(n=$ 256), the overall response rate (ORR) and disease control rate (DCR) were 39.06 and $64.45 \%$, respectively. In previous phase II study, the AITL patients received chidamide have a higher ORR of $50 \%$. Higher ORR and superior survival were also observed for AITL patients received romidepsin and belinostat. In this real world study, AITL patients also tend to have higher ORR and DCR of $49.23 \%$ and $75.38 \%$ which were comparable with previous results. It has been reported that epigenetic regulation plays an important role in AITL pathogenesis, which may be relevant to more clinical benefits by HDAC inhibitors to AITL. The ORR and DCR seem higher for ALK+ ALCL patients receiving chidamide of $66.67 \%$ and $83.33 \%$, but only 13 ALK+ ALCL patients receiving chidamide were included in this study and ALK+ ALCL alone has a better prognosis than other subtypes. Given that HDAC inhibitors can impair DNA repair mechanisms, thereby inducing DNA damage, the effects of HDAC inhibitors may be synergistic with the effects of chemotherapy. Several studies have shown that HDAC inhibitors combined with chemotherapy constitute an efficient treatment for PTCL patients, yet the optimal combination regimen remains unknown. This study found that the ORR and DCR were 51.18 and $74.02 \%$, respectively, for patients receiving chidamide combined with chemotherapy $(n=127)$. For patients with an International Prognostic Index (IPI) of $2-3$, the ORR in the chidamide combined with chemotherapy group $(n=55)$ was $58 \%$ higher than that in the chidamide single-agent group $(n=141)$, with an ORR of $41 \%$ $(P=0.0031)$. Chidamide combined with chemotherapy also increased the ORR for patients with an IPI of 4-5 $(n=26)$ relative to the ORR of patients receiving chidamide alone $(n=40)$ with ORRs of 42 and $10 \%$, respectively $(P=0.006)$. The results of a subgroup analysis showed that the ORRs for patients receiving chidamide combined with cyclophosphamide, doxorubicin, vincristine, and prednisone (CHOP)-like regimens, platinumcontaining regimens, and other regimens were 53.13, 45.83 , and $55.32 \%$, respectively, with DCRs of 81.25 , 66.67 , and $76.60 \%$, respectively (Table 1 ).

For patients receiving chidamide monotherapy and chidamide combined with chemotherapy, the median progression-free survival (PFS) was 129 (95\% CI 82 to 194) days and 152 (95\% CI 93 to 201) days, respectively $(P=0.3266)$ (Fig. 1$)$ and the median duration of response (DOR) was 148 (95\% CI 132 to 171) days and 169 (95\% CI 154 to 192$)$ days, respectively $(P=0.3215)$. In the chidamide monotherapy group, the PFS for AITL and peripheral $\mathrm{T}$ cell lymphoma-not otherwise specified (PTCL-NOS) patients were 144.5 days and 133 days, respectively. In the combination group, the PFS for AITL and PTCL-NOS patients were 176 days and 124 days, respectively. The results of a subgroup analysis showed that the median PFS for patients receiving chidamide combined with CHOP-like regimens, platinum-containing regimens, and other regimens was 172,119 , and 160 days, respectively. The median DOR for patients receiving chidamide combined with CHOPlike regimens, platinum-containing regimens, and other regimens was 180,165 , and 172 days, respectively.

Drug-related adverse events (AEs) that occurred in $\geq 5 \%$ of patients receiving chidamide alone included thrombocytopenia (25.0\%), neutropenia (19.1\%), fatigue (18.4\%), nausea/vomiting (14.1\%), and anemia (11.3\%). Drug-related AEs that occurred in $\geq 5 \%$ of patients receiving chidamide combined with chemotherapy included thrombocytopenia (28.4\%), neutropenia (25.2\%), 


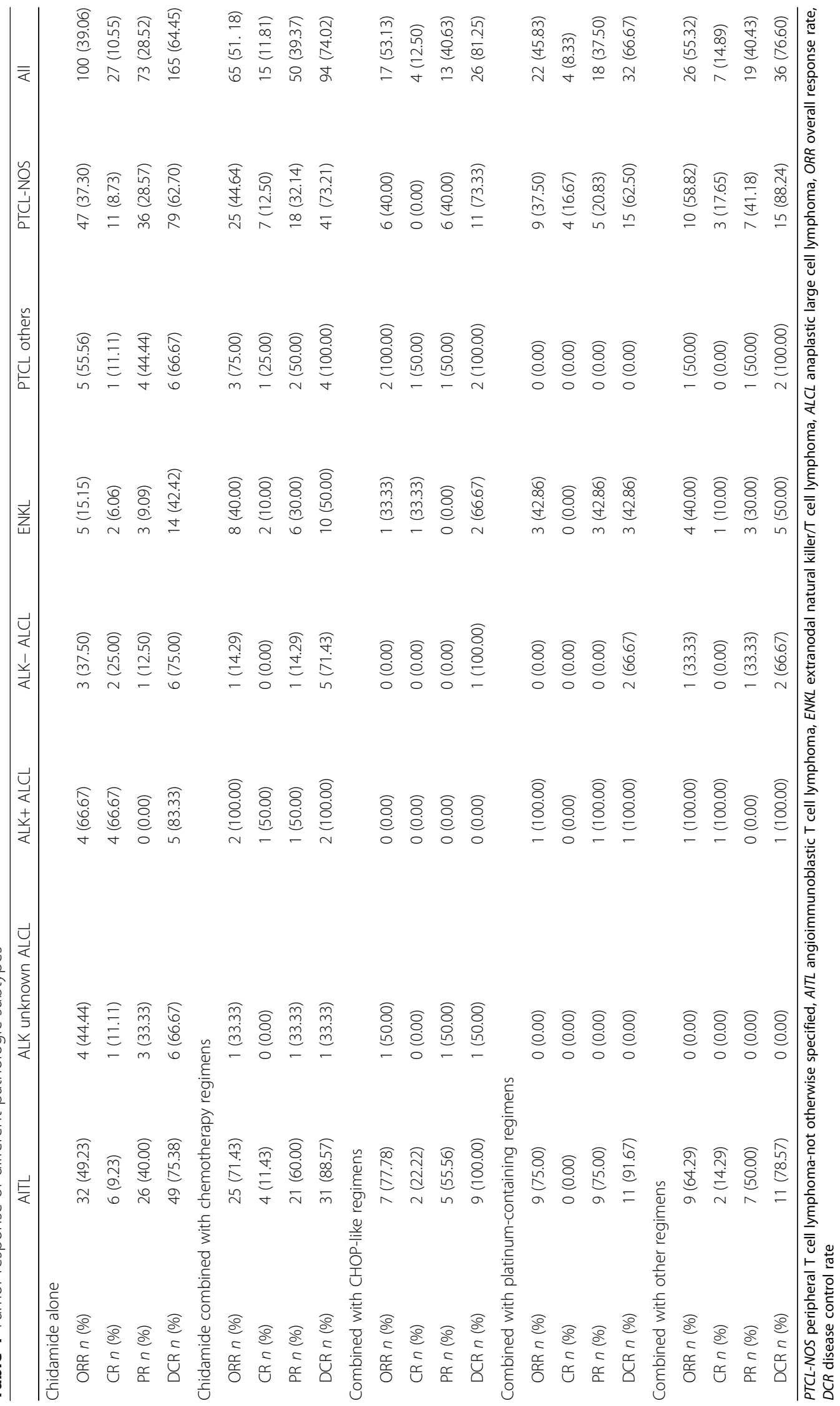




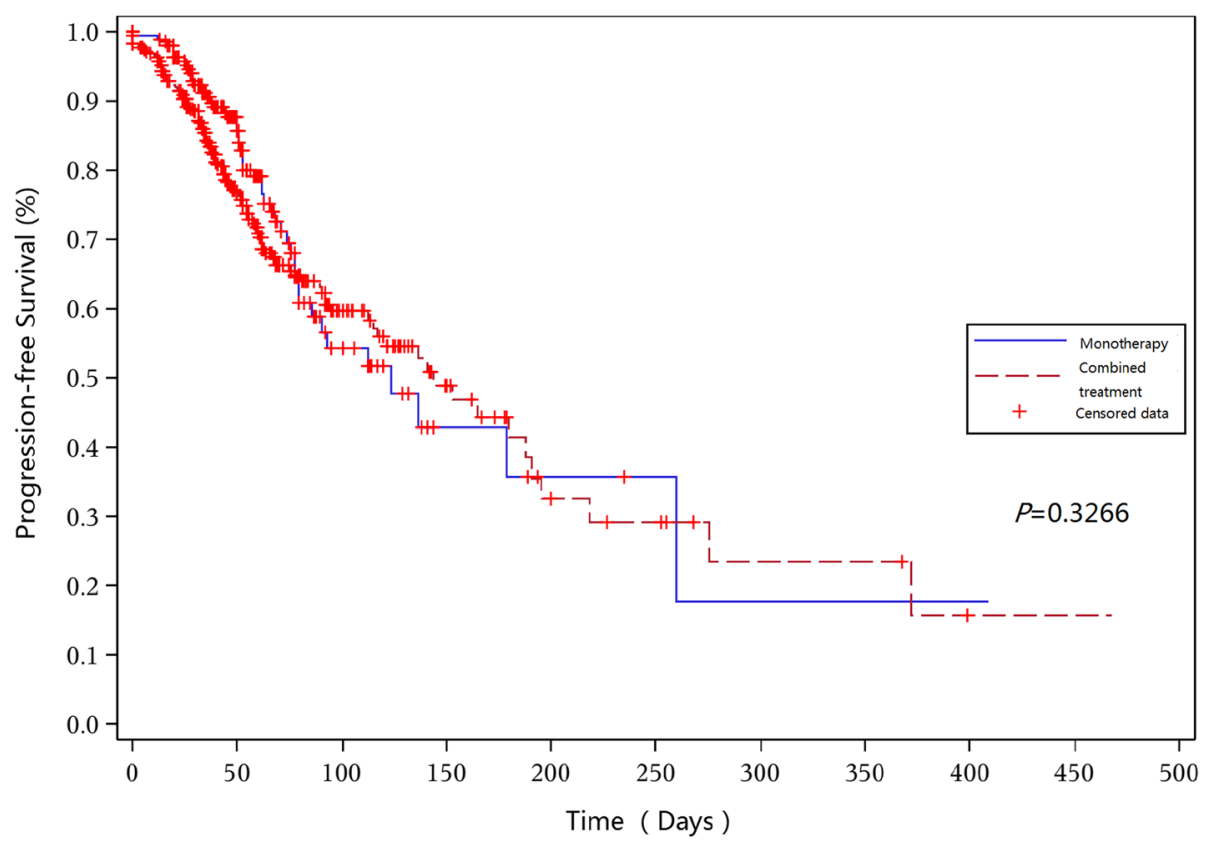

Fig. 1 Progression-free survival for patients receiving chidamide monotherapy and patients receiving chidamide combined with chemotherapy

fatigue (24.4\%), anemia (17.3\%), nausea/vomiting (12.7\%), increased alanine aminotransferase (ALT) (9.5\%), and increased aspartate aminotransferase (AST) (6.3\%). Most AEs were of grade 1 to 2 . AEs of grade 3 or higher that occurred in $\geq 5 \%$ of patients receiving chidamide alone included thrombocytopenia (10.2\%) and neutropenia (6.2\%). For patients receiving chidamide combined with chemotherapy, grade 3 to 4 AEs that occurred in $\geq 5 \%$ of patients included thrombocytopenia (18.1\%), neutropenia (12.6\%), anemia (7.1\%), and fatigue (5.5\%) (Additional file 3).

In summary, this real-world study conducted with 383 patients demonstrates that chidamide has a favorable efficacy and an acceptable safety profile for refractory and relapsed PTCL patients, confirming the pivotal phase II study in a more representative realworld population. Moreover, this study indicated the potential benefit of chidamide when combined with chemotherapy, which had not been previously examined. Chidamide combined with chemotherapy may be a new treatment choice for PTCL, especially for PTCL patients with an IPI $\geq 2$, although further investigation is warranted.

\section{Additional files}

Additional file 1: Methods (DOCX $16 \mathrm{~kb}$ )

Additional file 2: Table S1. Patients' baseline characteristics (DOCX $16 \mathrm{~kb}$ ) Additional file 3: Table S2. Drug-related adverse events in $\geq 5 \%$ of patients (DOCX $21 \mathrm{~kb})$
Abbreviations

AEs: Adverse events; ALT: Alanine aminotransferase; ASCT: Autologous stem cell transplantation; AST: Aspartate aminotransferase; CFDA: China Food and Drug Administration; DCR: Disease control rate; DOR: Duration of response; FDA: Food and Drug Administration; HDAC: Histone deacetylase; IPI: International Prognostic Index; NHL: Non-Hodgkin's lymphoma; ORR: Overall response rate; PFS: Progression-free survival; PTCL: Peripheral T cell lymphoma

\section{Acknowledgments}

The authors thank the patients, medical staff, and physicians who participated in this study. The authors thank Jun Ma, Jun Zhu, Jianyong Li, Zhixiang Shen, and Wenqi Jiang for their contribution to this study. The authors also acknowledge Meta Clinical Technology Co., Ltd. for the data analysis

\section{Funding}

This study was sponsored by Chipscreen Biosciences, Ltd., Shenzhen, China.

Availability of data and materials

The datasets supporting the conclusions of this article are included within the article and additional files.

\section{Authors' contributions}

YS contributed to the conception and design of this study. All authors contributed to the provision of the patients in this study, the collection of data, and the writing of the manuscript. All authors read and approved the final manuscript.

Competing interests

The authors declare that they have no competing interests.

\section{Consent for publication}

Not applicable.

\section{Ethics approval and consent to participate}

Chidamide was approved in December 2014 by the China Food and Drug Administration (CFDA) for the treatment of relapsed or refractory PTCL, and the treatment choice was made by oncologists in each center. All patients 
signed the chemotherapy informed consent before the treatment, and the anonymity of the patients has been maintained.

\section{Publisher's Note}

Springer Nature remains neutral with regard to jurisdictional claims in published maps and institutional affiliations.

\section{Author details}

'Department of Medical Oncology, Beijing Key Laboratory of Clinical Study on Anticancer Molecular Targeted Drugs, National Cancer Center/Cancer Hospital, Chinese Academy of Medical Sciences and Peking Union Medical College, Beijing 100021, China. ${ }^{2}$ Peking University Cancer Hospital and Institute, Beijing, China. ${ }^{3}$ Jiangsu Province Hospital, Nanjing, China. ${ }^{4}$ Guangdong General Hospital, Guangzhou, China. ${ }^{5}$ West China Hospital, Sichuan University, Chengdu, China. ${ }^{6}$ Zhongshan Hospital, Shanghai, China. ${ }^{7}$ Shanghai Ruijin Hospital, Shanghai, China. ${ }^{8}$ Tianjin Medical University Cancer Institute and Hospital, Tianjin, China. ${ }^{9}$ The Second Hospital of Dalian Medical University, Dalian, China. ${ }^{10}$ Zhejiang Cancer Hospital, Hangzhou, China. ${ }^{11}$ Xinqiao Hospital, Third Military Medical University, Chongqing, China. ${ }^{12}$ The First Affiliated Hospital, Zhejiang University, Hangzhou, China. ${ }^{13}$ The First Affiliated Hospital of Soochow University, Suzhou, China. ${ }^{14}$ Sun Yat-Sen University Cancer Center, Guangzhou, China. ${ }^{15}$ Hematology Institute and Hospital, Chinese Academy of Medical Sciences and Peking Union Medical College, Tianjin, China. ${ }^{16}$ Sichuan Provincial People's Hospital, Chengdu, China. ${ }^{17}$ Hunan Cancer Hospital, Changsha, China. ${ }^{18}$ Shandong Provincial Hospital, Jinan, China. ${ }^{19}$ Jiangsu Cancer Hospital, Nanjing, China. ${ }^{20}$ Nanjing Drum Tower Hospital, Nanjing, China. ${ }^{21}$ Tongji Hospital, Wuhan, China. ${ }^{22}$ Fujian Provincial Cancer Hospital, Fuzhou, China. ${ }^{23}$ The First Affiliated Hospital of Chongqing Medical University, Chongqing, China. ${ }^{24}$ Union Hospital, Fujian Medical University, Fuzhou, China. ${ }^{25}$ General Hospital of Nanjing Military Region, Nanjing, China. ${ }^{26}$ Changhai Hospital, Shanghai, China. ${ }^{27}$ The First Affiliated Hospital of Anhui Medical University, Hefei, China.

${ }^{28}$ Sichuan Cancer Hospital and Institute, Chengdu, China. ${ }^{29}$ Xijing Hospital, The Fourth Military Medical University, Xi'an, China. ${ }^{30}$ Anhui Provincial Hospital, Hefei, China. ${ }^{31}$ QiLu Hospital of Shandong University, Jinan, China. ${ }^{32}$ Liaoning Cancer Hospital and Institute, Dalian, China. ${ }^{33}$ Nanfang Hospital, Southern Medical University, Guangzhou, China. ${ }^{34}$ The 307th Hospital of Chinese People's Liberation Army, Beijing, China. ${ }^{35}$ Fourth Hospital of Hebei Medical University (Tumor Hospital of Hebei Province), Shijiazhuang, China. ${ }^{36}$ The Second Hospital of Hebei Medical University, Shijiazhuang, China. ${ }^{37}$ Shanxi Provincial Cancer Hospital, Taiyuan, China. ${ }^{38}$ The First Affiliated Hospital of Guangzhou Medical University, Guangzhou, China. ${ }^{39}$ Tianjin People's Hospital, Tianjin, China. ${ }^{40}$ Beijing Tongren Hospital, Beijing, China.

${ }^{41}$ The First Affiliated Hospital of Harbin Medical University, Harbin, China.

${ }^{42}$ Peking University Shenzhen Hospital, Shenzhen, China. ${ }^{43}$ The Second Affiliated Hospital Zhejiang University School of Medicine, Hangzhou, China. ${ }^{44}$ Peking Union Medical College Hospital, Beijing, China. ${ }^{45}$ The First Hospital of Jilin University, Changchun, China. ${ }^{46}$ Wuhan Union Hospital of China, Wuhan, China.

Received: 16 January 2017 Accepted: 7 March 2017

Published online: 15 March 2017

\section{References}

1. Vose J, Armitage J, Weisenburger D. International peripheral T-cell and natural killer/T-cell lymphoma study: pathology findings and clinical outcomes. J Clin Oncol. 2008;26(25):4124-30.

2. Yang QP, Zhang WY, Yu JB, Zhao S, Xu H, Wang WY, et al. Subtype distribution of lymphomas in Southwest China: analysis of 6,382 cases using WHO classification in a single institution. Diagn Pathol. 2011;6(1):77.

3. Sun J, Yang Q, Lu Z, He M, Gao L, Zhu M, et al. Distribution of lymphoid neoplasms in China: analysis of 4,638 cases according to the World Health Organization classification. Am J Clin Pathol. 2012;138(3):429-34.

4. Jia B, Hu S, Yang J, Zhou S, Liu P, Qin Y, et al. Comparison of gemcitabin, cisplatin, and dexamethasone (GDP), CHOP, and CHOPE in the first-line treatment of peripheral T-cell lymphomas. Hematology. 2016;21(9):536-41.

5. Jung KS, Cho SH, Kim SJ, Ko YH, Kang ES, Kim WS. L-asparaginase-based regimens followed by allogeneic hematopoietic stem cell transplantation improve outcomes in aggressive natural killer cell leukemia. J Hematol Oncol. 2016;9(1):1-4.
6. Gui L, Shi YK, He XH, Lei YH, Zhang HZ, Han XH, et al. High-dose therapy and autologous stem cell transplantation in peripheral T-cell lymphoma: treatment outcome and prognostic factor analysis. Int J Hematol. 2014;99(1):69-78.

7. O'Connor OA, Pro B, Pinter-Brown L, Bartlett N, Popplewell L, Coiffier B, et al. Pralatrexate in patients with relapsed or refractory peripheral T-cell lymphoma: results from the pivotal PROPEL study. J Clin Oncol. 2011;29(9):1182-9.

8. Coiffier B, Pro B, Prince HM, Foss F, Sokol L, Greenwood M, et al. Results from a pivotal, open-label, phase II study of romidepsin in relapsed or refractory peripheral T-cell lymphoma after prior systemic therapy. J Clin Oncol. 2012;30(6):631-6.

9. Lu X, Ning Z, Li Z, Cao H, Wang X. Development of chidamide for peripheral T-cell lymphoma, the first orphan drug approved in China. Intractable Rare Dis Res. 2016;5(3):185-91.

10. Shi $Y$, Dong M, Hong $X$, Zhang W, Feng J, Zhu J, et al. Results from a multicenter, open-label, pivotal phase II study of chidamide in relapsed or refractory peripheral T-cell lymphoma. Ann Oncol. 2015;26(8):1766-71.

\section{Submit your next manuscript to BioMed Central and we will help you at every step:}

- We accept pre-submission inquiries

- Our selector tool helps you to find the most relevant journal

- We provide round the clock customer support

- Convenient online submission

- Thorough peer review

- Inclusion in PubMed and all major indexing services

- Maximum visibility for your research

Submit your manuscript at www.biomedcentral.com/submit
) Biomed Central 